\title{
Balk Properties of Syndiotactic 1,2-Polybutadiene. III. Melting and Crystallization Properties
}

\author{
Yutaka OвAтA, Chikara Homma, and Chikao Tosaki \\ Research Laboratory, Japan Synthetic Rubber Co., \\ Mochiizaka, Ikuta, Tama-ku, Kawasaki, Japan.
}

(Received July 5, 1974)

\begin{abstract}
The Melting and crystallization behavior of 1,2-polybutadiene with 25-\% crystallinity were observed by DSC thermograms at various heating and cooling rates. The effects of annealing were observed through melting phenomena of the sample, at temperature from 40 to $95^{\circ} \mathrm{C}$.

It was found that the specimen annealed for a long time at a fixed temperature had two endothermic peaks in its melting thermogram, one corresponding to the annealing temperature and the other to the melting of the crystal formed at the stage of annealing at room temperature or in the quenching period after the annealing. An analytical equation was proposed for the kinetics of crystallization at constant cooling rate on the bases of Avrami's equation. The proposed relation appeared to be valid over a wide range of $x / x_{\infty}$ not only for the present sample but also for other crystalline polymers such as isotactic polypropylene. This method enables us to observe crystallization behavior at lower temperature side than that by dilatometry. The characteristics of the kinetics of 1,2-polybutadiene were as follows. The induction time for the crystallization was relatively large and the temperature coefficient of the rate of crystallization was small as compared to those of highly crystalline polymers such as high- and lowdensity polyethylenes, and isotactic polypropylene.
\end{abstract}

\section{KEY WORDS 1,2-Polybutadiene / DSC / Annealing / Kinetics of Crystallization /}

In the previous paper $^{1}$, the outline of the melting behavior of syndiotactic 1,2-polybutadiene was discussed in relation to the degree of crystallinity. The present study was undertaken for industrial purposes to obtain the information necessary for processing by technique such as extrusion, blow molding and to elucidate aging effects during storage at room temperature. In such processing, the molten specimen is cooled rapidly and the crystallization and melting behavior in such a state may well be observed by DSC thermogram under a wide range of cooling and heating rates.

Effects of annealing on the endothermic thermograms have been investigated for crystalline polymers, in relation to the multi-endothermic peaks which have been discussed from various points of view. ${ }^{2}$ The identification of the endothermic peaks and the estimation of the equilibrium melting temperature may then be the most important precursors which should be performed prior to the observation of the thermogram in melting and crystallization behavior. Previous investigations showed that the melting thermogram of 1,2-polybutadiene with different degrees of crystallinity has two endothermic peaks. The lower peak temperature was found to be between 45 and $50^{\circ} \mathrm{C}$ and was independent of the degree of crystallinity $x$ of the sample, while the second peak temperature varied with $x$. Further, the heat of fusion of crystal estimated from the total area of the endothermic peaks was nearly proportional to $x$.

The present investigation was based on these results.

Investigation of the kinetics of crystallization has been performed by the dilatometric method under isothermal conditions and the theory has been well established. ${ }^{3,4}$ The DSC technique ${ }^{7}$ has been also used with a small test specimen which allows the heat generated by the crystallization to diffuse quickly. 


\section{PREPARATION OF TEST SPECIMEN}

Specimens of syndiotactic 1,2-polybutadiene with $25-$ and $46-\%$ crystallinity designated by S25 and S46, ${ }^{1}$ isotactic polypropylene (iso-PP) and high- and low-density polyethylene (HD-PE, LD-PE) were press-molded by the following successive processes; five minutes preheating, ten minutes press at $300 \mathrm{~kg} / \mathrm{cm}^{2}$, and 5 -min cooling by water. The molding temperatures of S25, S46, iso-PP, HD-PE, and LD-PE were 160, 200, 180,180 , and $180^{\circ} \mathrm{C}$, respectively.

Before measurement, the molded specimens were annealed at room temperature for more than a week to obtain a reference state for the thermal history. Certain molecular characteristics of S25 and S46 were reported in the previous paper. $^{1}$

\section{MELTING BEHAVIOR}

\section{Identification of Endothermic Peaks}

In order to identify the endothermic peaks of the sample S25 having the reference thermal history, the following DSC measurement was designed. The test specimen of about $20 \mathrm{mg}$ was first cooled down to $-80^{\circ} \mathrm{C}$ at a rate of $40^{\circ} \mathrm{C} / \mathrm{min}$, and then heated up to $160^{\circ} \mathrm{C}$ at a constant heating rate of $16^{\circ} \mathrm{C} / \mathrm{min}$ (stage I), then cooled again at a constant cooling rate of $16^{\circ} \mathrm{C} /$ min (stage $\mathrm{II}$ ), and heated again at the same heating rate as stage I (stage III). The differential power input was scanned all through the stages. The thermograms in stages I, II, and III of S25 are shown in Figure 1. The melting endotherm in stage $\mathrm{I}$, which is the thermogram of the specimen having the reference thermal history, has two peaks, while the exotherm and endotherm in stages II and III are both unimodal. Considering the fact that the specimen is in a completely molten state at the end of stage $I$, the difference in the melting thermograms in stages I and III may be attributed to the difference in their thermal histories. Further it was found that the heat of fusion, $\Delta H_{\mathrm{f}}$, in stage $\mathrm{I}$ and the heat of crystallization $\Delta H_{\mathrm{c}}$ in stage II were identical for all of the 1,2polybutadiene samples reported in the previous paper, as shown in Figure 2. These phenomena may lead as to conclude that a part of the

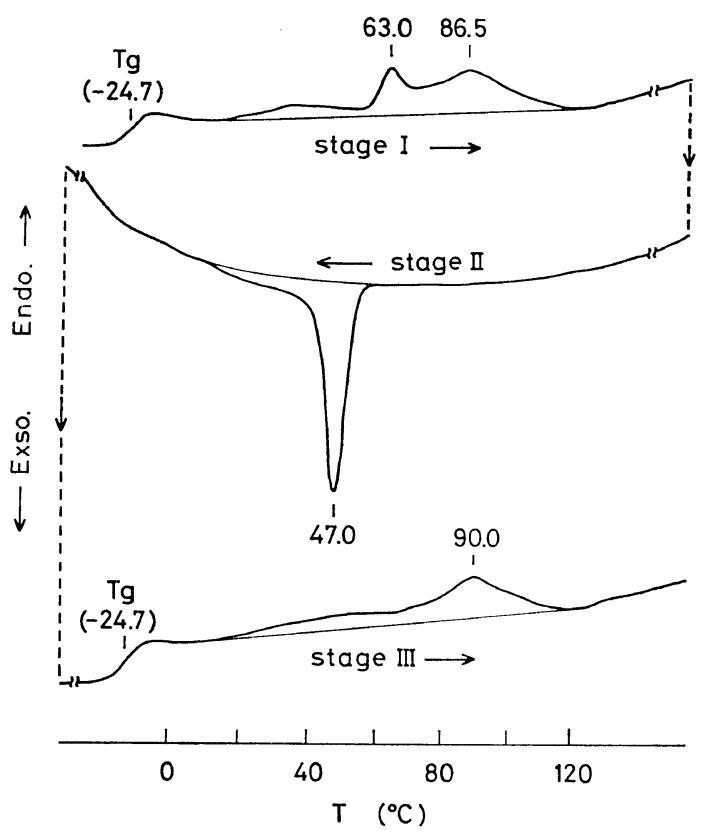

Figure 1. DSC thermogram of sample $\mathbf{S} 25$ scanned at $20^{\circ} \mathrm{C} / \mathrm{min}$. The specimen having the reference thermal history was quenched to $-80^{\circ} \mathrm{C}$ and $\mathrm{DSC}$ thermogram was obtained in heating, cooling and heating processes in a succesive manner.

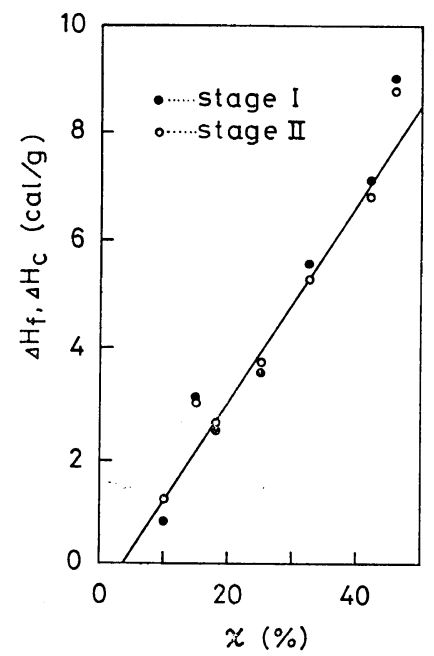

Figure 2. Heat of fusion $\Delta H_{\mathrm{f}}$ and $\Delta H_{\mathrm{c}}$ observed in stages I and II, respectively, of 1,2-polybutadiene samples with different degree of crystallinity at $30^{\circ} \mathrm{C}$. 
crystal produced in a stage II recrystallizes to form a new crystal structure in the course of annealing at room temperature. This conclusion will be further supported by the analysis for the data on annealing effects in the following paragraph.

\section{Effects of Annealing}

Among factors affecting the annealing effects, the annealing temperature and annealing time may be most important. In this experiment, the effects of both factors were examined. The effects of annealing time appeared to become time-independent when the specimen was annealed for more than $30 \mathrm{~min}$. The effects of temperature at a fixed annealing time of $30 \mathrm{~min}$ are shown below.

The testing specimen sealed within an aluminum pan was melted at $160^{\circ} \mathrm{C}$ in an oil bath for $10 \mathrm{~min}$ to reach a completely molten state, quenched in a methanol-dry ice system for a sufficient time and annealed at its respective temperature for $30 \mathrm{~min}$ in an oil bath and quenched again in the methanol-dry ice system. Several test pieces annealed at various temperatures were, thus, prepared. The aluminum pan was then picked up and dried at $25^{\circ} \mathrm{C}$ for $10 \mathrm{~min}$ and the specimen put into the testing apparatus where the DSC thermogram in the melting stage was scanned at a heating rate of $20^{\circ} \mathrm{C} / \mathrm{min}$. In the above processes, it should be noticed that the specimen has been crystallized in both annealing and quenching stages as well as in the stage of annealing at $25^{\circ} \mathrm{C}$. Figure 3 shows the thermograms of the specimens annealed at different temperatures ranging from 40 to $95^{\circ} \mathrm{C}$. The thermogram changes systematically with the change in annealing temperature in a way that an endothermic peak appears for each sample at a temperature 10 to $15^{\circ} \mathrm{C}$ higher than the respective annealing temperature. On the other hand the terminal temperature of melting $\left(T_{\mathrm{e}}\right)$ appeared to be nearly independent of the annealing temperature. The thermograms of the specimens annealed at temperatures higher than $50^{\circ} \mathrm{C}$ shows another endothermic peak corresponding either to the quenching or to the annealing stage at $25^{\circ} \mathrm{C}$. Further, the heat of fusion $\Delta H_{\mathrm{f}}$ was found to be independent of the annealing temperature.

These results indicate that the structure of the

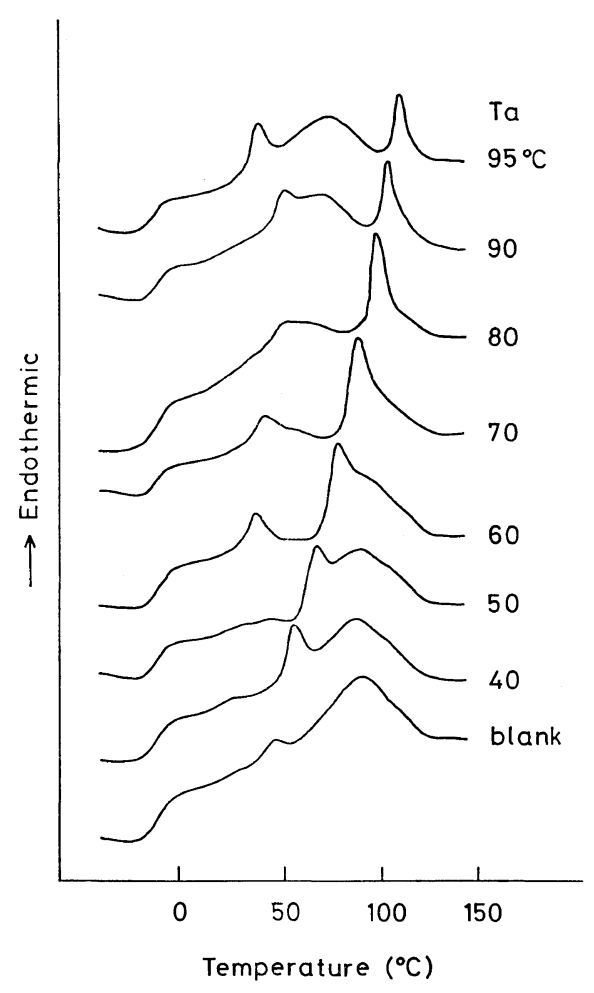

Figure 3. DSC thermograms of sample S25 annealed at various temperatures for $30 \mathrm{~min}$. The thermograms were scanned at $20^{\circ} \mathrm{C} / \mathrm{min}$.

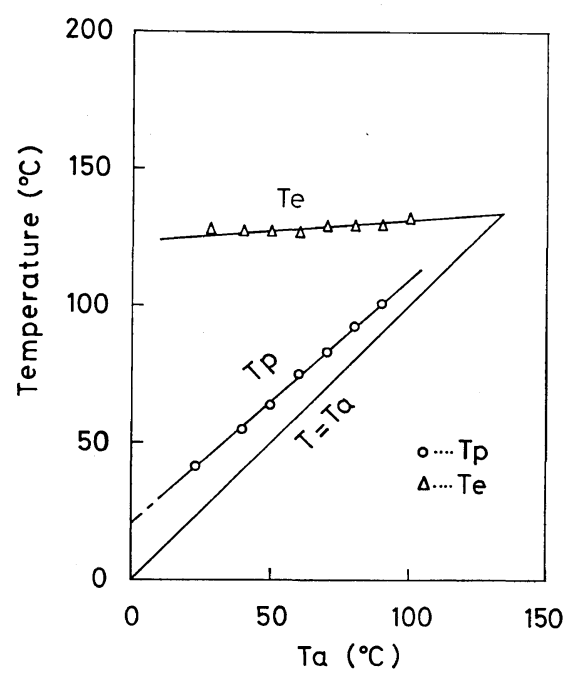

Figure 4. Temperatures at terminal point of melting $\left(T_{\mathrm{e}}\right)$ and at the endothermic peak corresponding to the annealing $\left(T_{\mathrm{p}}\right)$. 
crystal attained at the end of annealing melts partially and recrystallizes again during the long annealing time at room temperature to form a modified crystal structure.

The peak temperatures corresponding to the annealing temperature $\left(T_{\mathrm{p}}\right)$ are plotted against the corresponding annealing temperatures $\left(T_{\mathrm{a}}\right)$ in Figure 4. In the figure, the point where $T_{\mathrm{e}}$ equal to $T_{\mathrm{a}}$ is $133^{\circ} \mathrm{C}$. This temperature may be higher than the true equilibrium melting temperature because of superheating effects. The superheating effects in $\mathbf{S 2 5}$ in the reference state was measured by means of the thermograms scanned at different heating rates ranging from 2 to $64^{\circ} \mathrm{C} / \mathrm{min} . T_{\mathrm{e}}, T_{\mathrm{p}}$ and the peak temperature at a lower temperature region around $45^{\circ} \mathrm{C}\left(T_{1}\right)$ are plotted against the heating rate $(\alpha)$ in Figure 5. The extrapolation of $T_{e}$

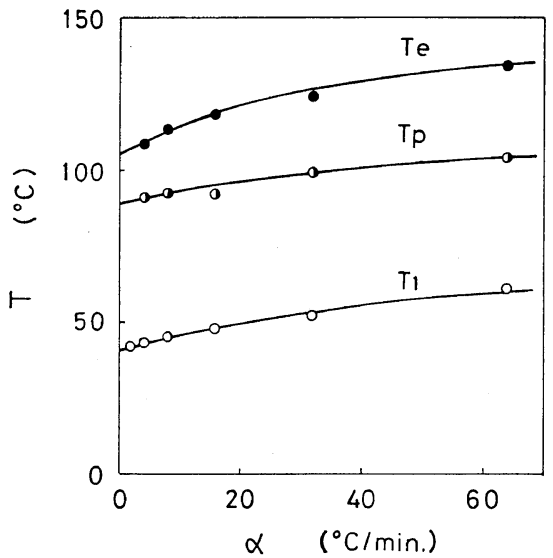

Figure 5. Dependency of the thermogram on the scanning rate, expressed by $T_{\mathrm{e}}, T_{\mathrm{p}}$, and $T_{1}$ which is the peak temperature at lower temperature side; S25 having reference thermal history.

towards $\alpha=0$ gives $114^{\circ} \mathrm{C}$ and it is about $15^{\circ} \mathrm{C}$ lower than $T_{\mathrm{e}}$ at $\alpha=20^{\circ} \mathrm{C} / \mathrm{min}$. It is therefore estimated that the value at the equilibrium melting temperature would fall in the temperature range from 110 to $118^{\circ} \mathrm{C}$.

\section{KINETICS OF CRYSTALLIZATION}

\section{Theory}

Avrami and other workers ${ }^{3,4,6}$ have established a general formula for the kinetics of isothermal crystallization such that

$$
\begin{aligned}
& \frac{\mathrm{d} x}{x_{\infty}-x}=\frac{\nu(t, \tau) N^{\prime}(\tau) \mathrm{d} \tau}{x_{\infty}} \\
& \nu(t, \tau)=G(t-\tau)^{n}
\end{aligned}
$$

where $x$ and $x_{\infty}$ denote the degree of crystallinity at time $t$ and at the final state, respectively. $N^{\prime}$ $(\tau)$ is the nucleation frequency per unit of the uncrystallized volume, $\nu(t, \tau)$ stands for the volume of a growing center at time $t$, which was originated at time $\tau,(\tau \leqq t)$ and $G$ is the growth rate constant. The parameter $n$ is an integer indicating the dimension of crystal growth.

At the nonisothermal state where the temperature decreases linearly with time, $N^{\prime}(\tau)$ in the above equation would generally be a function of temperature. Generally $x_{\infty}$ is also a function of temperature. The temperature dependence of $x_{\infty}$, however, was neglected to avoid complexity. The following assumption was made for the temperature dependence of nucleation rate $N^{\prime}(\tau)$ and the growth rate $G(\tau)$,

$$
N^{\prime}(T) G(T)=\gamma \exp (-\beta T)
$$

where $\gamma$ and $\beta$ are parameters and $T$ is the absolute temperature of the system at time $t$.

The crystallization time $t$ is defined by the following equation with the temperature at which crystallization begins $\left(T_{i}\right)$ and the cooling rate $\alpha$.

$$
t \equiv \frac{T_{i}-T}{\alpha}
$$

Substituting eq 2 and 3 into eq 1 and integrating on $\tau$ and $x$ side by side, an equation for the kinetics of crystallization under constant cooling rate is derived.

$$
\ln \frac{x_{\infty}}{x_{\infty}-x}=\frac{\gamma}{x_{\infty}} \exp \left(-\beta T_{i}\right) \int_{0}^{t} \exp (\alpha \beta \tau)(t-\tau)^{n} \mathrm{~d} \tau
$$

The integration for the right-hand side of the equation can be performed as follows:

$$
\begin{aligned}
& I_{n}=\int_{0}^{t} \exp (\alpha \beta \tau)(t-\tau)^{n} \mathrm{~d} \tau \\
& I_{0}=\frac{\exp (\alpha \beta t)-1}{\alpha \beta} \\
& I_{n}=\frac{n I_{n-1}-t^{n}}{\alpha \beta}, \quad n=1,2,3
\end{aligned}
$$

As is easily seen in the second equation of eq 5 , 


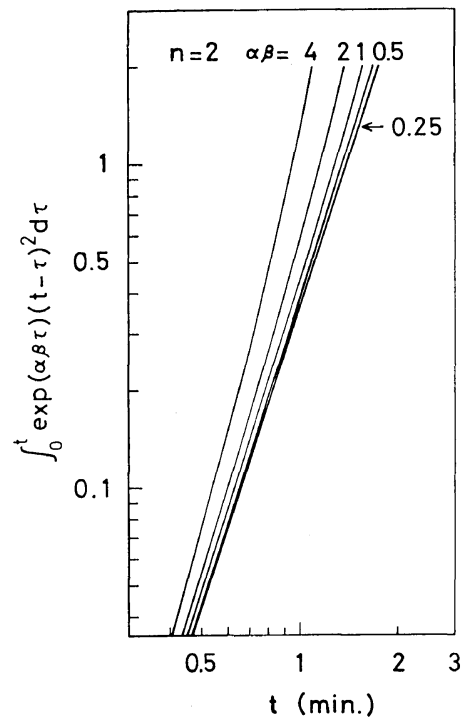

Figure 6. Calculated curves of $\int_{0}^{t} \exp (\alpha \beta \tau)(t-\tau)^{2} \mathrm{~d} \tau$ for various values of parameters $\alpha \beta$.

$I_{0}$, becomes independent of $\alpha \beta$, as $\alpha \beta$ approaches zero. Figure 6 shows the calculated curves of $\log I_{2} v s . \log t$ for various values of $\alpha \beta$. The results show that $I_{2}$ can be approximated by a power function of $t$, and it can be shown that this could be generalized for $I_{n}$ to give

$$
I_{n}=C(n, \alpha \beta) t^{n^{\prime}}
$$

Equation 6 is essentially the same formula as that Avrami has proposed, except that $n^{\prime}$ is not equal to $n+1$. Equation 4 is then approximated to give

$$
\begin{aligned}
\ln \frac{x_{\infty}}{x_{\infty}-x} & \doteqdot \frac{\gamma C}{x_{\infty}} \exp \left(-\beta T_{i}\right) t^{n^{\prime}} \\
& \equiv K_{\mathrm{s} n} t^{n^{\prime}}
\end{aligned}
$$

It should be noticed that eq 7 is only applicable to the cases where the assumption expressed by eq 2 is valid and $\alpha \beta$ is not very large.

Values of $I_{n}$ as a function of $t$ were calculated by eq 7 over a range of $0.04<I_{n}<0.8$ and values of $n^{\prime}$ are plotted against $\alpha \beta$ for $n=1,2,3$ in Figure 7. Figure 7 shows that $n^{\prime}$ approaches $n+1$ as $\alpha \beta$ approaches to zero.

Another interesting aspect of the kinetics of crystallization is the temperature dependence of the rate of crystallization. It can be well characterized by the inverse of time $t$ at $x / x_{\infty}=0.5$.

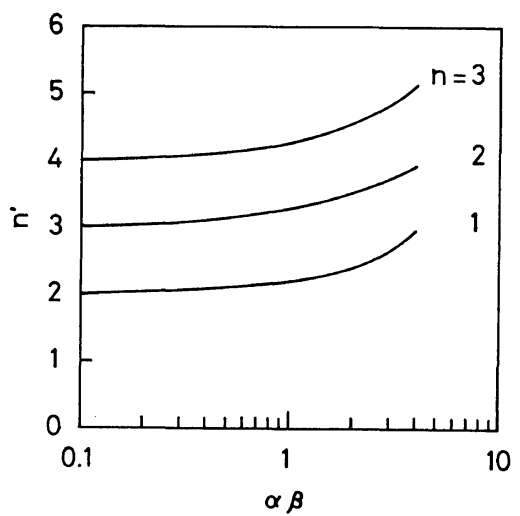

Figure 7. Calculated values of $n^{\prime}$ as a function of $\alpha \beta$ for values of $n$ expressing the dimension of crystal growth.

By replacing $t_{1 / 2}$ and $T_{1 / 2}$ respectively in eq 7 , the inverse of $t_{1 / 2}\left(1 / t_{1 / 2}\right)$ which is a measure of the rate of crystallization is given by

$$
\frac{1}{t_{1 / 2}}=\left\{\frac{\gamma C}{x_{\infty} \ln 2} \exp \left(-\beta T_{1 / 2}\right)\right\}^{1 / n}
$$

Equation 8 gives the relation between the rate and $T_{1 / 2}$. In a particular case where $T_{i}$ is transformed to $T_{1 / 2}$ (temperature at $t=t_{1 / 2}$ ) by a linear relation such that

We have

$$
T_{1 / 2}=a+b T_{i}
$$

$$
\frac{1}{t_{1 / 2}}=\left\{\frac{\gamma C}{x_{\infty} \ln 2}\right\}^{1 / n^{\prime}} \exp \left(\frac{-a \beta}{n^{\prime}}\right) \exp \left(\frac{-b \beta T_{i}}{n^{\prime}}\right)
$$

If the conditions expressed by eq 2 and 10 are satisfied, eq 8 and 10 predict that $\ln \left(t_{1 / 2}\right) v s$. $T_{1 / 2}$ or $T_{i}$ is a linear relationship, and the slopes are $\beta / n^{\prime}$ and $b \beta / n^{\prime}$, respectively.

\section{Experiments for Crystallization Behavior}

When the test specimen is cooled from the molten state at a fixed cooling rate, the thermogram exhibits an exothermic peak as seen in Figure 1. In the exothermic peak, if the ordinate is eliminated the contribution of the base line, it gives the rate of exothermic heat $\mathrm{d} H_{\mathrm{c}} / \mathrm{d} t$ due to the crystallization, which may be assumed to be proportional to the rate of crystallization $\mathrm{d} x / \mathrm{d} t$. The degree of crystallinity $x(T)$ at temperature $T$, is then, given by 
Bulk Properties of Syndiotactic 1,2-Polybutadiene. III.

$$
x(T)=K \int_{T}^{T_{i}}\left(\mathrm{~d} H_{\mathrm{c}} / \mathrm{d} t\right) \mathrm{d} T \equiv K^{\prime} A(T)
$$

where $T_{i}$ is the temperature where the crystallization begins, $K$ and $K^{\prime}$ are proportionality constants and $A(T)$ is the area of the exotherm integrated between $T_{i}$ and $T$. In the following analysis, a normalized variable $x / x_{\infty}$ will be used and it is defined by

$$
x / x_{\infty}=A(T) / A_{\infty}
$$

Here $A_{\infty}$ is the total area of the exothermic peak given by the integration over the entire crystallization temperature range.

Another problem is that the crystallization process is generally divided into at least two portions corresponding to the primary and secondary crystallizations. The latter may arise at the terminal stage as can be seen in the exothermic peak in Figure 1. The effects, however, were neglected in drawing the base line. The effects due to neglecting the secondary crystallization were estimated to be small for the data of S25. But this is not generally the case for the other crystalline polymers, which will be discussed later.

As an example of the dependence of the exothermic peak on the cooling rate, the exothermic peaks of S25 without the contribution of the base line are plotted against temperature (not against the scanning time $t$ ) in Figure 8 . It is observed that the value of $T_{i}$ becomes lower as the cooling rate $\alpha$ becomes larger; namely, the crystallization process accompanies an induction period. The values of temperature $T_{i}$ of $\mathrm{S} 25$ and other samples were obtained from Figure 8 and others, and they are plotted against the cooling rate in Figure 9. The relation between $T_{i}$ and $\alpha$ is linear except for a region of $\alpha$ less than $4^{\circ} \mathrm{C} / \mathrm{min}$. Denoting the intercept at the ordinate by $T_{i 0}$, the negative value of the slope

$$
\frac{T_{i 0}-T_{i}}{\alpha} \equiv t_{\text {ird }}
$$

is defined as a measure of the induction period. It should be noted that $T_{i 0}$ is fairly low comparing to the melting temperature of the sample. (For S25, $T_{i 0}$ is $83^{\circ} \mathrm{C}$ and extrapolated value of $T_{\mathrm{e}}$ at $\alpha=0$ is $114^{\circ} \mathrm{C}$.) The parameter $t_{\text {ind }}$, however, is an invariant in a sense that it is

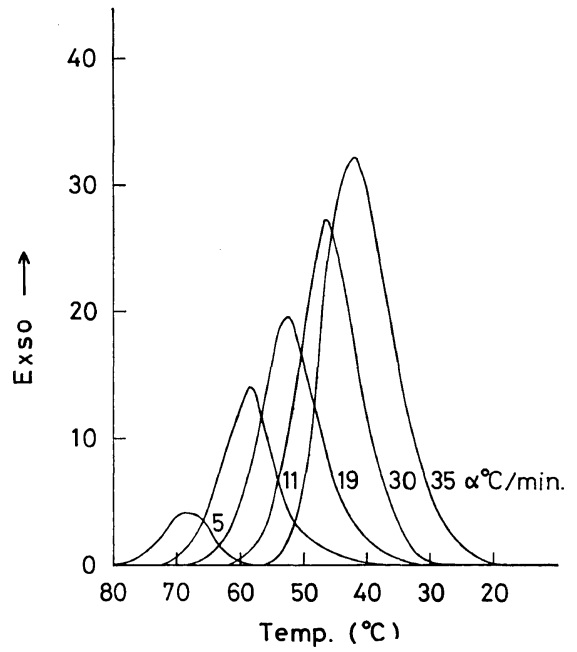

Figure 8. Exothermic peaks measured at different cooling rates plotted as a function of temperature.

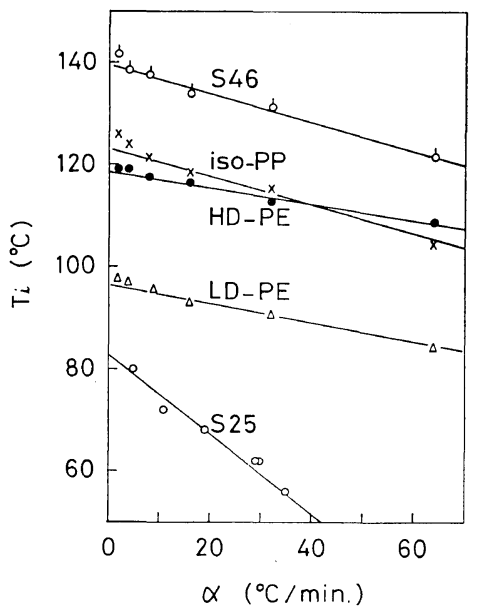

Figure 9. Temperature at the start of crystallization $T_{i}$ as a function of cooling rate for 1,2polybutadiene samples $\mathbf{S} 25$ and S46, and some highly crystalline polymers.

independent of $\alpha$. From Figure 9, it is noted that $t_{\text {ind }}$ of S25 is much larger than those of S46, HD-PE, LD-PE, and iso-PP.

To illustrate the temperature dependence of the rate of crystallization on the crystallization temperature, the plots of $1 / t_{1 / 2}$ against $T_{1 / 2}$ of S25 and other samples are shown in Figure 10. It is observed from the figure that $1 / t_{1 / 2}$ of $\mathbf{S} 25$ and $\mathrm{S} 46$ can be approximated by an exponentially decreasing function of $T_{1 / 2}$, namely the condition 


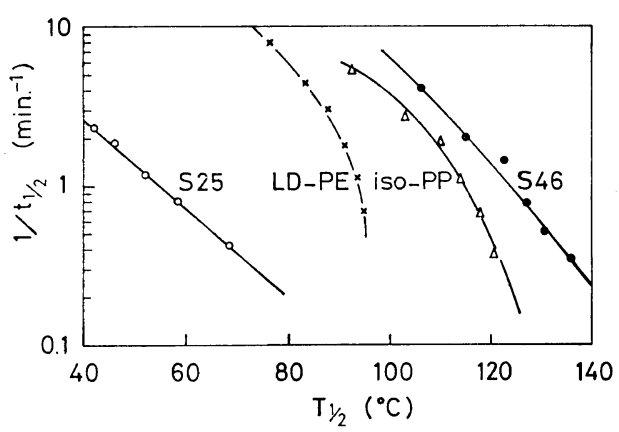

Figure 10. $1 / t_{1 / 2}$ (a measure of crystallization rate) as a function of temperature $T_{1 / 2}$.

expressed by eq 10 is satisfied for the samples over the temperature range examined, whereas it is not the case for other polymers of a higher degree of crystallinity. It should also be cautioned that the crystallization behavior was observed for a temperature region with a higher crystallization rate, i.e., at a lower temperature region than that for dilatometry. As an example, the observation for linear polyethylene can be made in the temperature range between $120^{\circ} \mathrm{C}$ and $130^{\circ} \mathrm{C}$ by means of dilatometry, while it is observed to be below $120^{\circ} \mathrm{C}$ as is shown in Figure 9. It is also noticeable that temperature dependence of the rate either for S25 or S46 is smaller than those of other highly crystalline polymers.

In Figures 11 and 12, values of $\left.\ln \left[\ln \left(x_{\infty} / x_{\infty}-x\right)\right)\right]$ are plotted as a function of $\log t$ for the data

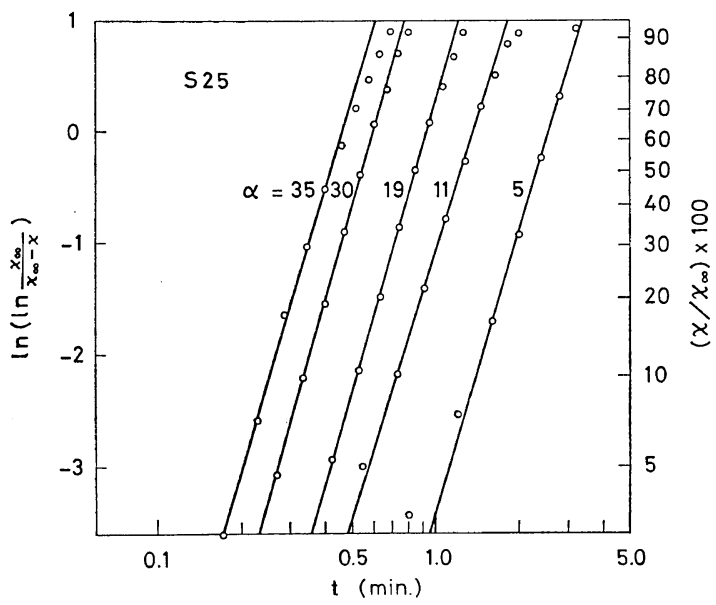

Figure 11. Avrami's plots of S25 for the data of crystallization at constant cooling rates $\alpha$.

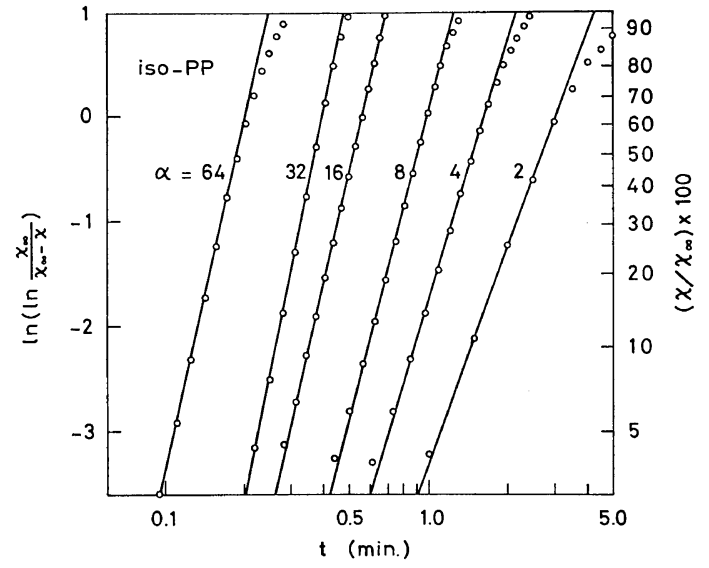

Figure 12. Avrami's plots of iso-PP for the data of crystallization at constant cooling rates $\alpha$.

Table I. Values of $n^{\prime}$ with the Cooling Rate

\begin{tabular}{rcrcrc}
\hline \multicolumn{2}{c}{ S25 } & \multicolumn{2}{c}{ S46 } & \multicolumn{2}{c}{ iso-PP } \\
\hline$\alpha^{\text {a }}$ & $n^{\prime}$ & $\alpha^{\text {a }}$ & $n^{\prime}$ & $\alpha^{\text {a }}$ & $n^{\prime}$ \\
\hline 5 & 3.16 & 4 & 2.60 & 4 & 3.43 \\
11 & 3.10 & 8 & 2.76 & 16 & 4.05 \\
19 & 3.15 & 16 & 2.78 & 16 & 4.77 \\
30 & 3.34 & 32 & 3.51 & 32 & 5.44 \\
35 & 3.26 & 64 & 3.25 & 64 & 4.62 \\
\hline
\end{tabular}

a ${ }^{\circ} \mathrm{C} / \mathrm{min}$.

on both S25 and iso-PP. The figures show that the Avrami's plot can also be applied to the analysis of the crystallization under various constant cooling rates within a range of $x / x_{\infty}$ from 0.05 to 0.8 . The deviation of the plots from the linearity which appears at the terminating portion of the crystallization may be ascribed to neglecting the effects of secondary crystallization. The values of $n^{\prime}$ in eq 7 were obtained from the slopes of the lines in Figures 10 and 11 and from those of the data of S46, which are listed in Table I with the values of corresponding cooling rates.

It is seen from the table that $n^{\prime}$ increases with increasing $\alpha$, and values of $n^{\prime}$ of S25 and S46 are relatively small comparing to that of iso-PP.

\section{DISCUSSIONS}

From the observation of the melting thermograms of the sample S25 annealed at different

Polymer J., Vol. 7, No. 3, 1975 
Bulk Properties of Syndiotactic 1,2-Polybutadiene. III.

temperatures in the melting temperature range, we may conclude that the occurrence of the multi-peaks can be attributed to the thermal history that the specimen has experienced.

In the analysis of the kinetics under constant cooling rate, some assumptions were introduced. First, $x_{\infty}$ in eq 1 was defined as the degree of crystallinity attained at the termination of the thermogram, but it should be replaced by $x_{\infty}(T)$, an equiliblium value of $x$ at $T$, so long as the concept of "effective fraction of the mass" in Avrami's equation ${ }^{6}\left(x_{\infty}-x\right) / x_{\infty}$ in eq 1$)$ is exactly followed. It should also be noted that the kinetic equation can be expressed in alternative ways by selecting the type of nucleation (homogeneous, heterogeneous) or the type of growth process $\left(G(r-\tau)^{n}\right)$ even in the case of isothermal crystallization $^{6}$. Therefore it does not make sense to use any physical meaning for $n^{\prime}$ or $n$ estimated from the data.

Secondary, the exotherm may be divided into two parts corresponding to the primary and secondary crystallizations. The primary crystallization can be analysed in terms of eq 7. It is expected that the range of $x / x_{\infty}$ where the data is well approximated by eq 7 would be expanded for larger values of $x / x_{\infty}$ provided the contribution due to the secondary crystallization were eliminated somehow. This result indicates that the Avrami's plot $\ln \left[\ln \left(x_{\infty} /\left(x_{\infty}-x\right)\right)\right] v s . \ln t$ is fairly insensitive to the change in cooling history, although the value of $n^{\prime}$ varies considerably with the change in cooling history. Kinetics of crystallization under given cooling history $T(\tau)$ is expected to be approximated by putting the $N^{\prime}(\tau) G(\tau)$ as a function of unique variable $T$. Then the constitutive equation for the kinetics can be written in the form

$$
\begin{aligned}
& \frac{\mathrm{d} x}{x_{\infty}-x}=\frac{1}{x_{\infty}} f(T)(t-\tau)^{n} \mathrm{~d} \tau \\
& f(T(\tau))=N^{\prime}(\tau) G(\tau) .
\end{aligned}
$$

Here $f(T)$ can be estimated from experiments.

Acknowledgment. The authors wish to express sincere thanks to Misses Fusako Shimizu, and Yoshiko Akiyama for their technical assistance in carrying out the experiments. The authors also acknowledge the helpful comments made by Drs. Terutake Homma, Masahiro Niinomi, Nobuhide Shiraishi and Kazuhiko Ninomiya.

\section{REFERENCE}

1. Y. Obata, M. Ikeyama, and C. Tosaki, Polymer J., 7, 207 (1975).

2. K. Kamiide, Kobunshi Kagaku (Chem. High Polymers), 25, 532 (1968).

3. M. Avrami, J. Chem. Phys., 7, 1103 (1939); ibid., 8, 212 (1940).

4. U. R. Evans, Trans. Faraday Soc., 41, 365 (1945).

5. K. Kamiide and K. Fujii, Kobunshi Kagaku (Chem. High Polymers), 25, 155 (1968).

6. L. Mandelkern, "Crystallization of Polymers," McGraw-Hill, New York, N. Y. 1964.

7. K. Nakamura, K. Katayama, and T. Amano, J. Appl. Poly. Sci., 17, 1031 (1973).

8. Gordon M. Martin and L. Mandelkern, J. Appl. Phys., 34, 2312 (1963). 